\title{
双核开链茂铁咪唑受体的合成与阴离子识别研究
}

\author{
卓继斌 $a$ 晏希泉 ${ }^{a}$ 王小雪 ${ }^{a}$ 谢莉莉 $a, b$ 袁耀锋*,,$b$ \\ ( ${ }^{a}$ 福州大学化学学院 福州 350116) \\ $(b$ 能源与环境光催化国家重点实验室 福州 350002)
}

\begin{abstract}
摘要 通过二茂铁甲基咪唑 1 与不同的二溴代烷反应合成了一系列双核开链的茂铁咪唑受体 $3 \mathrm{a} \sim 3 \mathrm{e}$, 化合物 $3 \mathrm{e}$ 的晶体 结构表明分子间有着强的 $\mathrm{C}-\mathrm{H} \cdots \mathrm{F}$ 氢键作用. 利用电化学和核磁共振氢谱考察了受体 $3 \mathbf{a} \sim 3 \mathbf{e}$ 对不同常见阴离子的识 别能力. 电化学滴定实验表明受体 $\mathbf{3 a} \sim 3 \mathbf{e}$ 在乙腈溶液中均能很好地识别 $\mathrm{F}^{-}$. 核磁滴定证实了受体与不同阴离子之间氢 键作用及参与类型. Job 曲线表明受体分子与阴离子间形成 $1: 1$ 型的配合物，通过络合常数 $\left(K_{\mathrm{a}}\right)$ 的对比表明受体 $3 \mathrm{c}$ 对氟 离子的选择性识别能力最好.
\end{abstract}

关键词 二茂铁; 开链; 咪坐鎓; 合成; 阴离子识别

\section{Synthesis and Anion Recognition of Acyclic Ferrocene-Based Imidazole Receptors}

\author{
Zhuo, Jibin $^{a} \quad$ Yan, Xiquan $^{a} \quad$ Wang, Xiaoxue $^{a} \quad{\text { Xie, } \text { Lili }^{a} \quad \text { Yuan, Yaofeng }}^{*, a, b}$ \\ ( ${ }^{a}$ College of Chemistry, Fuzhou University, Fuzhou 350116) \\ ( ${ }^{b}$ State Key Laboratory of Photocatalysis on Energy and Environment, Fuzhou University, Fuzhou 350002)
}

\begin{abstract}
The design and synthesis of artificial receptors capable of binding and sensing anions have been widely explored in the field of host-guest chemistry. Herein, a series of acyclic ferrocene-based imidazolium receptors $\mathbf{3 a} \sim \mathbf{3 e}$ were designed and synthesized. All the receptors were prepared by reactions of 1-(ferrocenylmethylene)imidazole 1 with corresponding dibromides in solvent under refluxing, followed by anion exchanges with $\mathrm{NH}_{4} \mathrm{PF}_{6}$. The structures of all the products were determined by IR, ${ }^{1} \mathrm{H}$ NMR, ${ }^{13} \mathrm{C}$ NMR, MS and elemental analysis. The crystal structure 3e was confirmed by X-ray crystallography, and it was clear that molecules were linked by $\mathrm{C}-\mathrm{H} \cdots \mathrm{F}$ hydrogen bonds interactions. The interaction between these receptors and various anions was studied by electrochemical measurements and ${ }^{1} \mathrm{H}$ NMR spectroscopy. The receptors $\mathbf{3 a} \sim \mathbf{3 e}$ were proven to be highly selective and sensitive to $\mathrm{F}^{-}$. ${ }^{1} \mathrm{H}$ NMR titrations demonstrated that the receptors and anions form 1 : 1 complex, and the receptors displayed a strong $(\mathrm{C}-\mathrm{H})^{+} \cdots \mathrm{X}^{-}$in anion recognition. Receptor $\mathbf{3 c}$ was the most effective receptor to bind with fluoride ion compared to the others.
\end{abstract}

Keywords ferrocene; acyclic; imidazolium; synthesis; anion recognition

阴离子因其在化学、材料学、生物学及环境科学等 相关领域发挥极其重要的作用, 因此设计合成对特定阴 离子具有单一选择性的人工受体已成为超分子化学研 究热点之一 ${ }^{[1]}$. 氟离子作为半径最小的阴离子, 是人体 必需的微量元素, 然而不恰当地摄入会引起氟中毒, 因 此氟离子识别对医学、环境、食品科学都具有重要的意

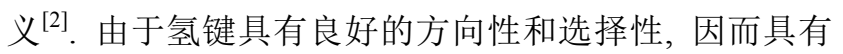
对氟离子形成强氢键的脉、硫䐂、酰胺、吡咯和吲哚等
活性基团的受体分子被陆续报道 ${ }^{[3]}$. 近年来，以咪唑鎓 主体构筑的带正电荷的阴离子受体引起广泛的关注 ${ }^{[4]}$. 该类受体具有强的静电作用，其 2 位酸性 $\mathrm{C}-\mathrm{H}$ 作为阴 离子识别位点, 可以与阴离子形成 $(\mathrm{C}-\mathrm{H})^{+} \cdots \mathrm{X}^{-}$这种离 子化的氢键, 具有氢键以及静电双重优点 ${ }^{[5]}$.

二茂铁具有氧化还原可逆性及易修饰性等诸多优 点，因其在电化学方面具有独特的优势，可作为良好的 电化学信号基团被引入人工受体中进行离子识别, 将茂

\footnotetext{
*E-mail: yaofeng_yuan@fzu.edu.cn
}

Received November 16, 2014; revised December 9, 2014; published online January 5, 2015.

Project supported by the National Natural Science Foundation of China (Nos. 21202019, 21372043), and the National Science Foundation for Fostering Talents in Basic Research of China (No. J1103303).

国家自然科学基金(Nos. 21202019, 21372043)、国家基础科学人才培养基金(No. J1103303)资助项目. 
铁基团引入咪唑受体中, 受体将具有咪唑及二茂铁双重 优点, 极大地丰富该类受体的电化学性质 ${ }^{[6]}$. 在阴离子 识别过程中, 阴离子与咪唑基团发生键合后, 茂铁的电 化学活性中心做出响应, 氧化峰电位向阳极或阴极移 动, 其移动大小代表了该受体对阴离子的应答水平 ${ }^{[7]}$. 人们通过监测受体分子的电位信号变化, 以实现对阴离 子的识别 ${ }^{[8]}$.

在前述基础上，我们合成了一系列双核开链茂铁咪 唑受体 3a 3e, 该类受体端基都含有阴离子识别单元 (咪唑)和传输识别过程的电化学信号单元(二茂铁). 借 助电化学手段, 通过阴离子与受体键合后导致受体构象 改变而得的电化学响应信号, 研究受体对不同阴离子的 亲和能力, 并利用核磁滴定实验进一步探究该类受体结 构与阴离子识别能力的关系. 结构表明含有双核开链的 茂铁咪唑受体对氟离子具有很好的选择性识别.

\section{1 结果与讨论}

\section{1 合成目标化合物研究}

目标化合物 $\mathbf{3 a} \sim \mathbf{3 e}$ 的合成路线如 Scheme 1 所示. 在二茂铁甲基咪唑 1 为原料与不同的二溴代烷反应生成 的双核茂铁咪唑溴盐 2 的过程中, 易得到 1,3-双二茂铁 基甲基咪唑溴盐 4. 该副产物形成的原因可能是因为溴 盐 2 在加热回流下存在两种共振式, 二茂铁具有很强的 富电子能力，长时间地加热易将化合物 2 中的甲基与咪 唑相连的化学键发生断裂形成稳定的二茂铁甲基正离 子 5 , 该正离子很容易与原料 1 反应形成副产物 $\mathbf{4}^{[9]}$. 因
此反应投料应控制二茂铁甲基咪唑与二溴代烷的比例 为 $2: 1$, 且在稀释的溶剂中避光反应, 从而避免副产物 的生成.

\section{2 目标产物的波谱特征}

通过溴盐 $2 \mathbf{a} \sim 2 \mathrm{e}$ 和六氟磷酸盐 $\mathbf{3 a} \sim \mathbf{3 e}$ 的 ${ }^{1} \mathrm{H}$ NMR 和 ${ }^{13} \mathrm{C} \mathrm{NMR}$ 对比发现: 改变受体分子的阴离子并不影 响其 NMR 中 $\mathrm{H}$ 和 $\mathrm{C}$ 的化学位移, 受体咪唑活泼氢化学 位移在 $\delta 8.98 \sim 9.14$, 碳的化学位移在 $\delta 135 \sim 136$, 其数 值与文献报道的咪唑盐类似 ${ }^{[10]}$. 同时对 $\mathbf{3 a} \sim 3 \mathbf{e}$ 活泼氢 化学位移进行对比, 可以发现随着两端基间的链增长, 活泼氢化学位移逐渐向低场移动, $\mathbf{3 a}$ 活泼氢化学位移的 化学位移在 $\delta 8.98$, 而 $3 \mathrm{e}$ 出现在 $\delta 9.14$, 这表明链的增 长使得受体的活泼氢更显酸性 ${ }^{[11]}$. 在 $-E S I$ full MS 负离 子模式下能明显的观测到化合物 $3 \mathbf{a} \sim \mathbf{3 e}$ 的 $145 \mathrm{PF}_{6}^{-}$离 子峰, 因此可以说明化合物 $\mathbf{2 a} \sim \mathbf{2 e}$ 在加入 $\mathrm{NH}_{4} \mathrm{PF}_{6}$ 后已 经转化为化合物 $\mathbf{3 a} \sim \mathbf{3 e}$. 同时 $\mathbf{3 a} \sim \mathbf{3 e}$ 出现在 $830 \sim 840$ $\mathrm{cm}^{-1}$ 左右强且尖锐的红外吸收峰可进一步验证 MS 的 结论.

\section{3 受体分子 $3 e$ 的晶体结构描述}

通过扩散法得到受体分子 $\mathbf{3 e}$ 的晶体结构并采用 $\mathrm{X}$ 射线衍射对其进行分析(图 1), 该化合物 $\mathrm{C}_{34} \mathrm{H}_{40} \mathrm{~F}_{12}$ $\mathrm{Fe}_{2} \mathrm{~N}_{4} \mathrm{P}_{2}\left(M_{\mathrm{r}}: 906.34\right)$ 的 CCDC 为 1033711 , 属于三斜晶 系, $P 2_{1} / c$ 空间群, 晶胞参数: $a=0.97955(6) \mathrm{nm}, b=$ $1.01846(6) \mathrm{nm}, c=1.06947(7) \mathrm{nm}, V=0.89587(10) \mathrm{nm}^{3}$, $D_{\mathrm{c}}=1.680 \mathrm{~g} \cdot \mathrm{cm}^{-3}, Z=1, F(000)=462, \mu=0.8233 \mathrm{~nm}^{-1}$.
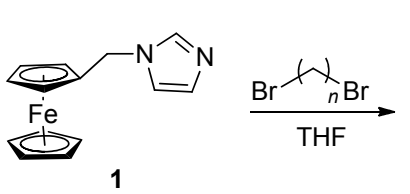

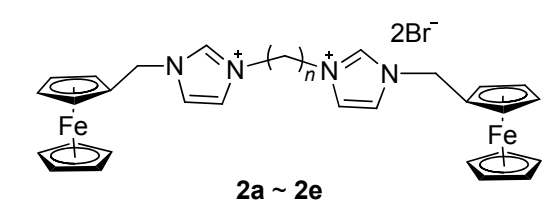

$n=2 \sim 6$

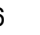

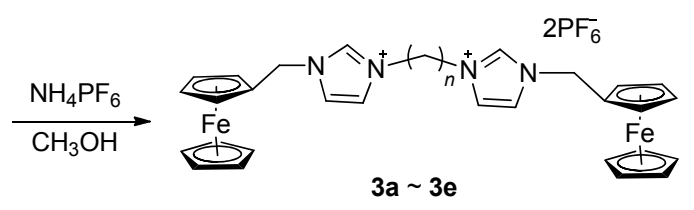

$3 a \sim 3 e$

图式 1 受体 $3 \mathrm{a} \sim 3 \mathrm{e}$ 的合成路线

Scheme 1 Synthetic routes of receptors $3 \mathbf{a} \sim 3 \mathbf{e}$
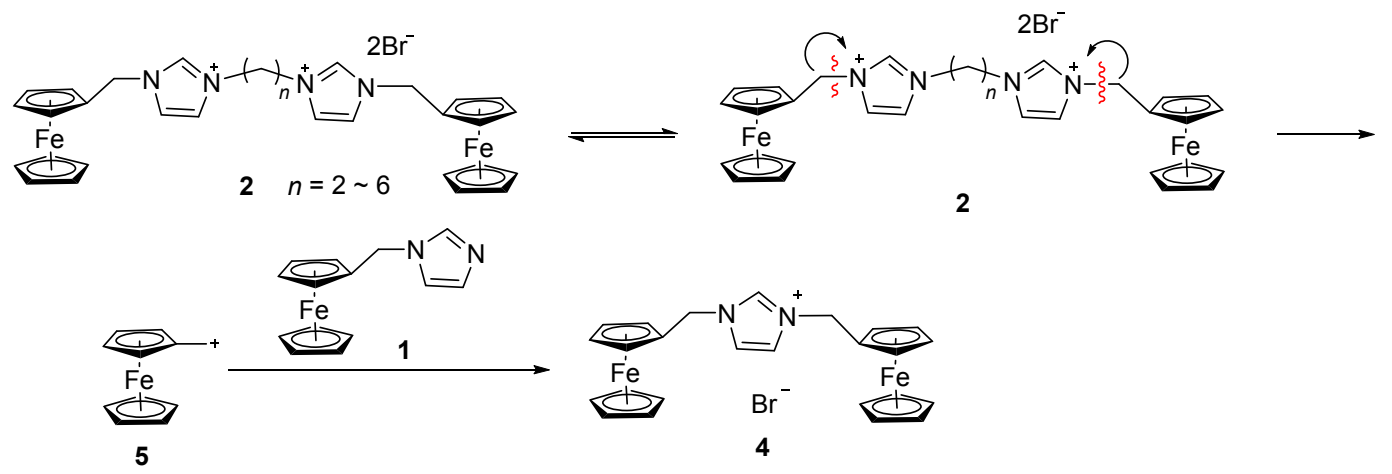

图式 2 化合物 2 的裂解机理

Scheme 2 Fragmentation mechanism of compound 2 


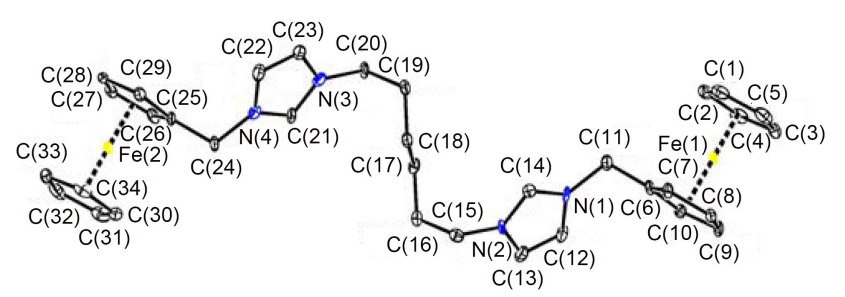

图 1 受体分子 $3 \mathrm{e}$ 的晶体结构

Figure 1 The crystal structure of receptor $3 \mathbf{e}$

根据受体分子 $3 \mathrm{e}$ 晶体结构及数据表明, 受体阳离 子构象基本属于中心对称, 两个茂铁中上下茂环互相平 行, 二面角分别为 $1.20^{\circ}$ 和 $1.81^{\circ}$, 两个二茂铁都处于重 叠式[扭曲角 $\mathrm{C}(1)-\mathrm{Cg}-\mathrm{Cg}-\mathrm{C}(6): 4.24^{\circ} ; \mathrm{C}(25)-\mathrm{Cg}-$ $\left.\mathrm{Cg}-\mathrm{C}(30): 4.98^{\circ}\right]^{[12]}$, 两个咪唑环与各自相邻茂铁 $\mathrm{Cp}$ 环 的夹角分别为 $71.37^{\circ}$ 和 $71.42^{\circ}$. 从图 2 可知, 分子式通过 $\mathrm{C}-\mathrm{H}-\mathrm{F}$ 氢键的形式相互作用连接(图 2). 因此受体阳 离子和六氟磷酸根阴离子通过氢键将其组装成了沿 $a$ 轴 无限延伸的超分子结构.

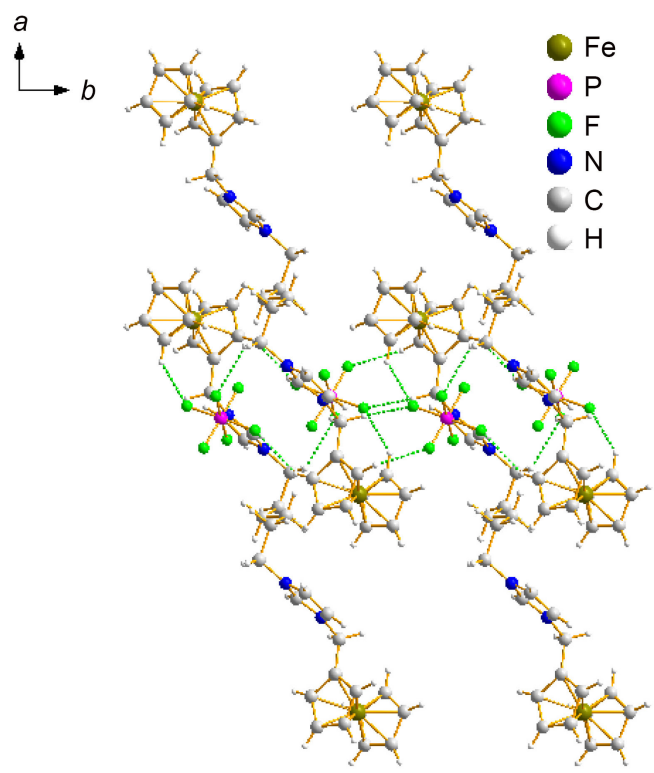

图 2 相邻分子之间的氢键作用

Figure 2 Hydrogen bonds between adjacent molecules of $\mathbf{3 e}$

\section{4 电化学滴定研究}

通过采用循环伏安法 $(\mathrm{CV})$ 研究了不同受体分子 3a $\sim 3 \mathrm{e}$ 及其与不同阴离子 $\left(\mathrm{F}^{-}, \mathrm{Cl}^{-}, \mathrm{Br}^{-}, \mathrm{I}^{-}, \mathrm{AcO}^{-}\right.$和 $\mathrm{HSO}_{4}^{-}$) 之间的相互作用, 乙腈溶液中, 当向受体分子 中滴加 $\mathrm{NBu}_{4} \mathrm{X}\left(\mathrm{F}^{-}\right.$和 $\left.\mathrm{AcO}^{-}\right)$时, 随着阴离子的滴加, 其 氧化峰逐渐增大并且还原峰逐渐消失, 氧化还原过程变 得不可逆(图 3a). 其原因可能是滴加的阴离子与受体结 合形成配合物 $\mathrm{R} \cdot \mathrm{X}^{-}$, 该配合物在电化学氧化后强烈地 吸附在了电极表面, 因此很难被还原 ${ }^{[13]}$. 接着采用差分 脉冲伏安法(DPV)监测了这两种阴离子对受体氧化峰电
位的影响，该方法在不可逆的体系中也能很好的观察其 氧化电位, 可以发现随着阴离子的滴加原先的氧化峰消 失, 在低电位处产生一个新的氧化峰. 这是由于这两种 阴离子与受体会产生较大的结合, 导致在滴定过程中受 体的茂铁基团存在于两种不同的环境中. 以 $3 \mathrm{c}$ 为例, 受 体对应于二茂铁基团的单电子氧化过程的 peak I 为 656 $\mathrm{mV}$ (图 3b), 滴加 $\mathrm{F}^{-}$使得该峰电位逐渐减低, 在 $486 \mathrm{mV}$ 产生一个新的峰电位 peak II, 两个电位的偏移值 $\Delta E=$ $178 \mathrm{mV} . \mathrm{AcO}^{-}$的滴加也会产生同样的现象, 然而其 $\Delta E$ 为 $32 \mathrm{mV}$, 明显小于 $\mathrm{F}^{-}$.
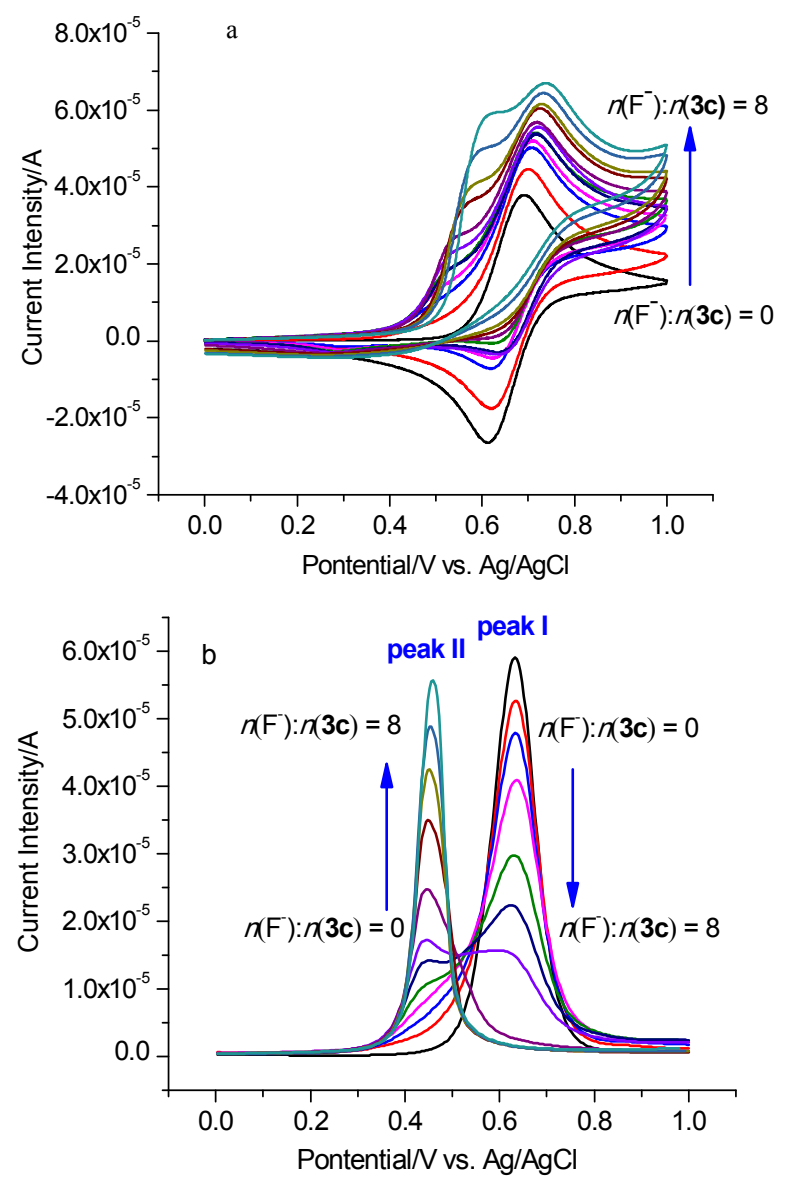

图 3 受体 $3 \mathrm{c}\left(c=10^{-3} \mathrm{~mol} \cdot \mathrm{L}^{-1}\right)$ 在乙腈溶液中对 $\mathrm{F}^{-}$识别的 $\mathrm{CV}$ (a) 和 DPV (b)曲线图

Figure $3 \mathrm{CV}$ (a) and DPV (b) of receptor $3 \mathbf{c}\left(c=10^{-3} \mathrm{~mol} \cdot \mathrm{L}^{-1}\right)$ in $\mathrm{CH}_{3} \mathrm{CN}$ with various concentrations of $\mathrm{F}^{-}$in the presence of $\left[n-\mathrm{Bu}_{4} \mathrm{~N}\right] \mathrm{PF}_{6}$

Scan rate: $100 \mathrm{mV} \cdot \mathrm{s}^{-1}$

当向受体 $3 \mathrm{c}$ 中滴加其他阴离子 $\mathrm{NBu}_{4} \mathrm{X}\left(\mathrm{X}=\mathrm{Cl}^{-}\right.$, $\mathrm{Br}^{-}, \mathrm{I}^{-}$和 $\mathrm{HSO}_{4}^{-}$), 可观察到 DPV 峰电位的负移动. 这 是因为受体与这些阴离子之间产生空间静电作用，使得 其电位向阴极移动, 然而这种静电作用较小, 因此电位 偏移量也较小. 根据其偏移量的大小, 受体 $\mathbf{3 c}$ 对不同阴 离子的电化学选择性顺序为 $\mathrm{F}^{-}>\mathrm{AcO}^{-} \approx \mathrm{HSO}_{4}^{-}>$ $\mathrm{Cl}^{-} \approx \mathrm{Br}^{-}>\mathrm{I}^{-}$. 其他不同链长的受体分子对不同阴离子 
的氧化电位峰偏移量列于表 1 . 从表 1 可以看出, 受体 分子 $3 \mathrm{a} \sim 3 \mathrm{e}$ 对 $\mathrm{F}^{-}$都有较大的响应. 随着链长的增加, 其对 $\mathrm{F}^{-}$的识别能力逐渐变强, 然而, 链增长到一定程 度, 对 $\mathrm{F}^{-}$的识别能力反而下降, 其原因可能是链长的增 加使得受体端基的两个识别基团能更好地包裹 $\mathrm{F}^{-}$, 但 是过长的链使得受体端基的两个基团共同结合 $\mathrm{F}^{-}$有一 定的困难. 同时, 链的增长也容易对不同体积的阴离子 (如 $\mathrm{AcO}^{-}$和 $\mathrm{HSO}_{4}^{-}$) 会有一定的影响, 随着双核识别基 团中间链长的增加, 受体对三角形结构的阴离子 $\left(\mathrm{AcO}^{-}\right)$ 的响应能力降低, 反而对四面体结构的阴离子 $\left(\mathrm{HSO}_{4}^{-}\right)$ 响应能力增加, 而大体积球形结构的阴离子 $\left(\mathrm{Br}^{-}\right.$和 $\left.\mathrm{I}^{-}\right)$ 的响应也会增加, 然而程度并不大, 这可能是由于链长 的增加导致受体过于柔性, 从而对大体积的阴离子都会 有一定的响应, 然而 $\mathrm{AcO}^{-}$由于其结构较小, 过长链的 受体其两端基的识别基团也难以同时对 $\mathrm{AcO}^{-}$进行结合. 综上所述, 受体中间链的增长会降低其对 $\mathrm{F}^{-}$的选择识 别, 通过比较, 受体 $3 \mathrm{c}$ 是一个理想的对氟离子有选择性 识别电化学受体.

表 1 受体 $3 \mathbf{a} \sim 3 \mathbf{c}$ 对不同阴离子识别的 $D P V$ 峰电位差 $(\mathrm{mV})$ 数据

Table $1 \Delta E_{\mathrm{p}}{ }^{a}$ values associated with the complexing processes between the free receptors and the appropriate anion ${ }^{b}$

\begin{tabular}{cccccccc}
\hline Receptor & $\mathrm{R}^{c}$ & $\mathrm{R} \cdot \mathrm{F}^{-b}$ & $\mathrm{R} \cdot \mathrm{Cl}^{-}$ & $\mathrm{R} \cdot \mathrm{Br}^{-}$ & $\mathrm{R} \cdot \mathrm{I}^{-}$ & $\mathrm{R} \cdot \mathrm{AcO}^{-}$ & $\mathrm{R} \cdot \mathrm{H}_{2} \mathrm{SO}_{4}^{-}$ \\
\hline 3a & 660 & -172 & -20 & -7 & -8 & -56 & -40 \\
3b & 656 & -178 & -15 & -7 & -13 & -36 & -44 \\
3c & 656 & -170 & $-11-12$ & -12 & -32 & -65 \\
3d & 658 & -152 & $-5-14$ & -16 & -23 & -70 \\
3e & 662 & -132 & $-5-18$ & -16 & -21 & -82 \\
\hline
\end{tabular}

${ }^{a} \Delta E_{\mathrm{P}}=E_{\text {complex }}-E_{\text {receptor. }}$ In millivolt, obtained from the DPV experiments carried out in $\mathrm{CH}_{3} \mathrm{CN} ;{ }^{b}$ anodic shifts upon 8 equiv. anion added in the form of the tetrabutylammonium salt; ${ }^{c} E_{\mathrm{p}}$ values corresponding to the free receptors.

\section{$1.5{ }^{1} \mathrm{H}$ NMR 滴定研究}

受体分子 $3 \mathbf{a} \sim 3 \mathbf{e}$ 对不同客体阴离子 $\left(\mathrm{F}^{-}, \mathrm{Cl}^{-}, \mathrm{Br}^{-}\right.$, $\mathrm{I}^{-}, \mathrm{AcO}^{-}$和 $\left.\mathrm{HSO}_{4}^{-}\right)$的亲和力通过核磁滴定实验进行考 察. 在 DMSO- $d_{6}$ 溶液中, 不同阴离子的滴加均引起咪唑 上活泼氢的低场偏移, 这是由于受体与阴离子形成了不 同强度 $(\mathrm{C}-\mathrm{H})^{+} \cdots \mathrm{X}^{-}$氢键和静电作用, 形成了 $\mathrm{R} \cdot \mathrm{X}$ 配合 物, 影响了其活泼氢的化学环境. 以 $3 \mathrm{c}$ 为例, 其咪唑上 活泼氢化学位移 $\left(\mathrm{H}_{\mathrm{a}}\right)$ 为 $\delta 9.13$, 咪唑环的另外两个氢化 学位移 $\left(\mathrm{H}_{\mathrm{b}}\right.$ 和 $\left.\mathrm{H}_{\mathrm{c}}\right)$ 分别为 $\delta 7.77$ 和 7.72, 取代 $\mathrm{Cp}$ 环的两 种氢化学位移 $\left(\mathrm{H}_{\mathrm{d}}\right.$ 和 $\left.\mathrm{H}_{\mathrm{e}}\right)$ 分别为 $\delta 4.44$ 和 4.26 , 未取代 $\mathrm{Cp}$ 的氢 $\left(\mathrm{H}_{\mathrm{f}}\right)$ 显示一个单峰, 化学位移为 $\delta 4.24$, 当加入等物 质的量的 $\mathrm{F}^{-}$后 $\mathrm{H}_{\mathrm{a}}$ 偏向低场移动至 $\delta 9.78$, 其偏移量 $\Delta \delta$ 为 $0.65 . \mathrm{H}_{\mathrm{b}}$ 和 $\mathrm{H}_{\mathrm{c}}$ 变成一个氢信号 $\delta 7.76$, 随着氟离子的 继续滴加, $\mathrm{H}_{\mathrm{a}}$ 信号变宽并且峰面积变小, 同时, $\mathrm{H}_{\mathrm{d}}$ 和 $\mathrm{H}_{\mathrm{e}}$ 信号也随着氟离子滴加而消失, 在 $\delta 16.3$ 处还出现了一 个 $\mathrm{HF}_{2}^{-}$的三重峰, 这是由于氟离子具有较大的电负性,
对氢具有去质子化效应. 然而 $\mathrm{H}_{\mathrm{f}}$ 和烷链上的氢没有太 大的变化(图 4). 其它阴离子 $\left(\mathrm{Cl}^{-}, \mathrm{Br}^{-}, \mathrm{I}^{-}, \mathrm{AcO}^{-}\right.$和 $\mathrm{HSO}_{4}^{-}$) 的滴加仅引起受体氢化学位移的偏移, 根据 $\mathrm{H}_{\mathrm{a}}$ 位移偏移量的大小: $\mathrm{F}^{-}>\mathrm{AcO}^{-}>\mathrm{Cl}^{-}>\mathrm{Br}^{-}>\mathrm{HSO}_{4}^{-} \approx$ $\mathrm{I}^{-}$, 可以看出 $3 \mathrm{c}$ 对氟离子具有很好的结合能力(图 5). 然而该结论与电化学有一点不同, 这是由于受体 $\mathbf{3 c}$ 与 $\mathrm{HSO}_{4}^{-}$在电化学过程中容易发生氢化反应 ${ }^{[14]}$, 而在 DMSO- $d_{6}$ 中, $\mathrm{Cl}^{-}$和 $\mathrm{Br}^{-}$负电荷密度比 $\mathrm{HSO}_{4}^{-}$高, 相比之 下更强的氢键作用导致了它们化学位移的偏移程度比 $\mathrm{HSO}_{4}^{-}$大.

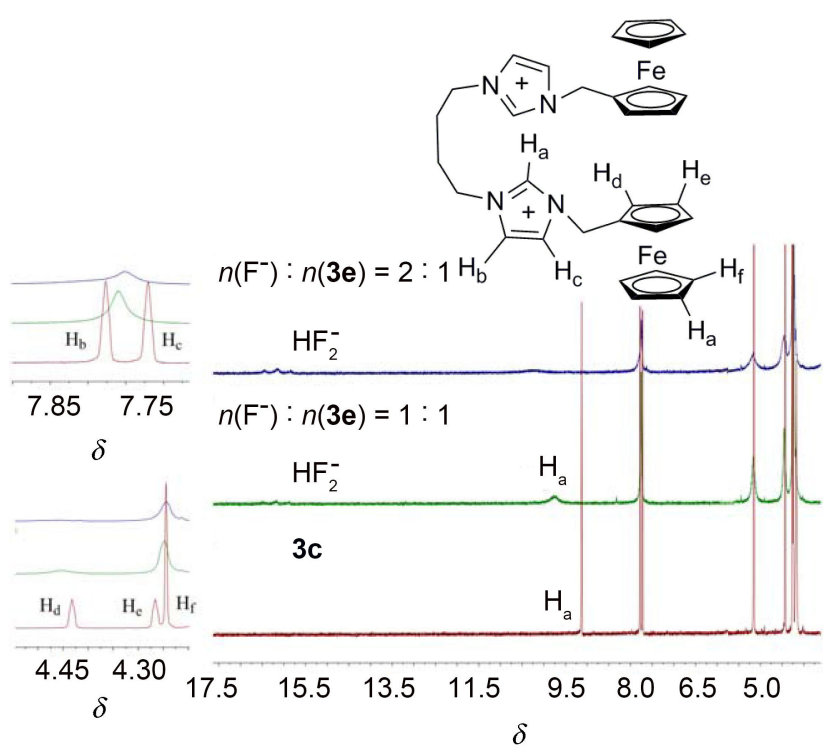

图 4 受体 3c 在 DMSO- $d_{6}$ 中加入不同浓度 $\mathrm{F}^{-}$的 ${ }^{1} \mathrm{H}$ NMR 堆 积图

Figure $4{ }^{1} \mathrm{H}$ NMR stack spectra of receptor $3 \mathbf{c}$ in DMSO- $d_{6}$ with various concentrations of $\mathrm{F}$

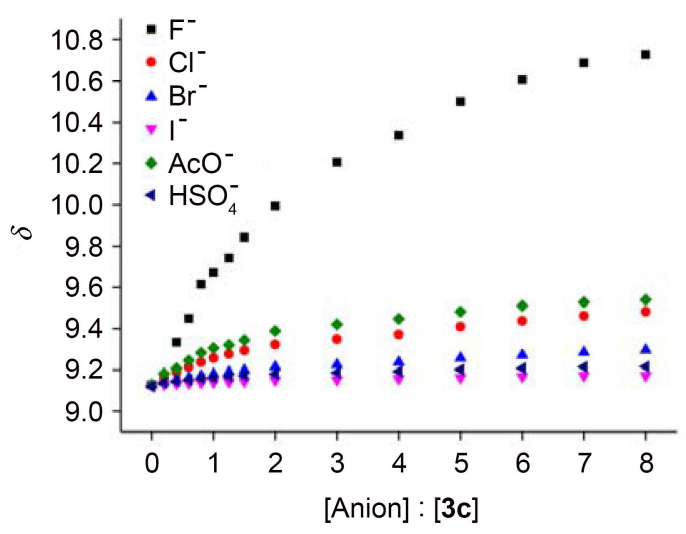

图 5 受体 $3 \mathrm{c}$ 在 DMSO- $d_{6}$ 中加入不同阴离子后通过监测咪唑 盐上 $\mathrm{C} 2$ 质子的 $1 \mathrm{H} \mathrm{NMR}$ 滴定曲线

Figure $5{ }^{1} \mathrm{H}$ NMR titration curves of receptor $3 \mathbf{c}$ in DMSO- $d_{6}$ with various concentrati ons of different anion by monitoring the $\mathrm{C} 2$ proton of imidazolium moieties

对于受体分子 (3a, 3b, 3d 和 3e), 不同阴离子对它们 活泼氢引起的位移偏移大小与 $\mathbf{3 c}$ 类似, 然而在加入等 
物质的量 $\mathrm{F}^{-}$时, 它们比 $3 \mathrm{c}$ 的偏移量略小, 该结论很好 地证明了咪唑中间链较短或者受体过于柔性都会影响 其对 $\mathrm{F}^{-}$的结合能力 (图 6). 以受体 $\mathbf{3 c}$ 为例, 通过监测其 咪唑活泼氢的位移变化研究主客体间的化学计量比和 络合常数, Job plot 表明主客体之间以 $1: 1$ 的比例作用 (图 7), 其络合常数列于表 2. 结果证明 $\mathbf{3 a} \sim 3 \mathbf{e}$ 对 $\mathrm{F}^{-}$均 有很好地结合, 其中, 受体 $3 \mathrm{c}$ 对 $\mathrm{F}^{-}$的选择性识别能力 最好.

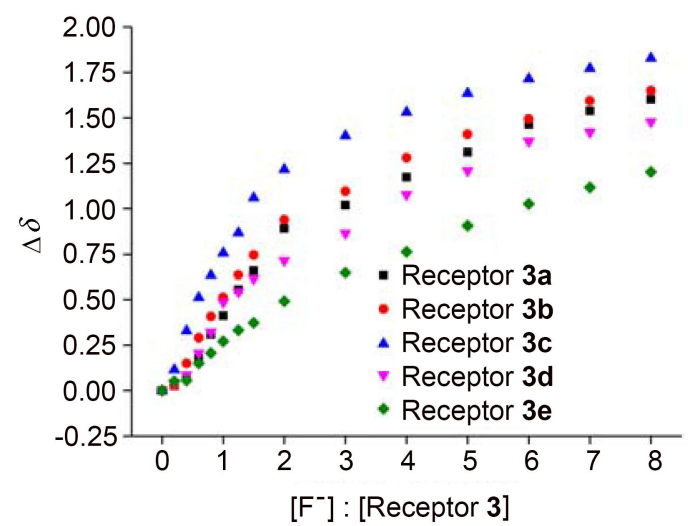

图 6 受体 3a $3 \mathrm{e}$ 在 DMSO- $d_{6}$ 中加入不同浓度 $\mathrm{F}^{-}$后通过监 测咪唑盐上 $\mathrm{C} 2$ 质子的 ${ }^{1} \mathrm{H}$ NMR 滴定曲线

Figure $6{ }^{1} \mathrm{H}$ NMR titration curves of receptors $\mathbf{3 a} \sim \mathbf{3 e}$ in DMSO- $d_{6}$ with various concentrations of $\mathrm{F}^{-}$by monitoring the $\mathrm{C} 2$ proton of imidazolium moieties

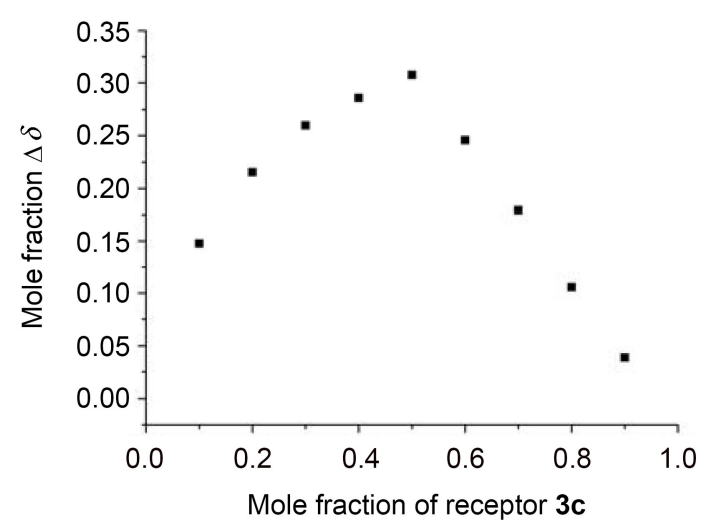

图 7 受体 $3 \mathrm{c}$ 在 DMSO- $d_{6}$ 中 $\mathrm{F}^{-}$的 Job 曲线

Figure 7 Job plots for receptor $3 \mathbf{c}$ with $\mathrm{F}^{-}$in DMSO- $d_{6}$

\section{2 结论}

设计合成了以咪唑单元为识别位点, 以二茂铁为电 化学信号的双核开链的茂铁咪唑受体 $3 \mathbf{a} \sim 3 \mathbf{e}$, 对其进 行了结构表征并考察了其对不同阴离子的识别作用. 受 体 3e 的单晶结构表明, 该受体分子通过分子间氢键作 用无限延伸形成超分子结构. 在电化学识别过程中, CV 曲线可以发现所有受体 $3 \mathrm{a} \sim 3 \mathrm{e}$ 在乙腈中对 $\mathrm{F}^{-}$有很好的 电化学响应, 在滴加 $\mathrm{F}^{-}$后均氧化峰增强, 还原峰消失的
表 2 受体 $3 \mathbf{a} \sim 3 \mathbf{c}$ 对不同阴离子的结合常数 ${ }^{a}$

Table 2 Association constants $\left(K_{a}\right)$ for $1: 1$ complexes of receptors 3a $\sim \mathbf{3 e}$ with anions in DMSO- $d_{6}$ at $298 \mathrm{~K}$

\begin{tabular}{lccccc}
\hline \multirow{2}{*}{ Anion $^{b}$} & \multicolumn{5}{c}{$K_{\mathrm{a}} /\left(\mathrm{mol} \cdot \mathrm{L}^{-1}\right)$} \\
\cline { 2 - 6 } & $\mathbf{3 a}$ & $\mathbf{3 b}$ & $\mathbf{3 c}$ & $\mathbf{3 d}$ & $\mathbf{3 e}$ \\
\hline $\mathrm{F}^{-}$ & $1.42 \times 10^{5}$ & $1.48 \times 10^{5}$ & $1.63 \times 10^{5}$ & $1.30 \times 10^{5}$ & $1.07 \times 10^{5}$ \\
$\mathrm{Cl}^{-}$ & $2.82 \times 10^{4}$ & $3.49 \times 10^{4}$ & $2.10 \times 10^{4}$ & $2.84 \times 10^{4}$ & $2.58 \times 10^{4}$ \\
$\mathrm{Br}^{-}$ & $1.51 \times 10^{3}$ & $1.74 \times 10^{3}$ & $1.50 \times 10^{3}$ & $1.56 \times 10^{3}$ & $1.36 \times 10^{3}$ \\
$\mathrm{I}^{-}$ & $2.83 \times 10^{2}$ & $3.10 \times 10^{2}$ & $3.90 \times 10^{2}$ & $3.90 \times 10^{2}$ & $4.70 \times 10^{2}$ \\
$\mathrm{AcO}^{-}$ & $6.84 \times 10^{3}$ & $7.47 \times 10^{3}$ & $3.68 \times 10^{3}$ & $3.78 \times 10^{3}$ & $2.23 \times 10^{3}$ \\
$\mathrm{HSO}_{4}^{-}$ & $7.74 \times 10^{2}$ & $7.83 \times 10^{2}$ & $7.92 \times 10^{2}$ & $1.78 \times 10^{3}$ & $1.93 \times 10^{3}$ \\
\hline${ }^{a}$ Errors are estimated to be $<1 \%^{6}{ }^{b}$ the tetrabutylammonium salts are em- \\
ployed as the source of anions.
\end{tabular}

现象，同时在 DPV 曲线可以发现原氧化峰消失且低电 位出现一个新的氧化峰, 实验表明受体 $\mathbf{3 a} \sim 3 \mathbf{e}$ 对 $\mathrm{F}^{-}$具 有很好地电化学选择性识别. 在 ${ }^{1} \mathrm{H}$ NMR 滴定实验中, $\mathrm{F}^{-}$的滴加能使咪唑上活泼氢变宽, 峰面积变小, 并且向 低场移动，同时在 $\delta 16.3$ 附近出现了一个 $\mathrm{HF}_{2}^{-}$的三重 峰，表明 $\mathrm{F}^{-}$与受体咪唑上的活泼氢形成静电作用和氢 键作用并对该氢产生去质子化. 其他位置的氢也因为氢 键作用而导致位移的改变或信号的消失，表明 $\mathrm{F}^{-}$对受 体其他位置的氢也有一定氢键作用，进一步证明受体分 子与阴离子间的氢键作用本质。阴离子实验表明，随着 双核开链的茂铁咪唑受体中间链长的增加，受体对 $\mathrm{F}^{-}$ 的识别能力先增强后减弱, 过长的柔性的受体也会对其 它结构的阴离子进行响应, 从而降低其对 $\mathrm{F}^{-}$的选择 性，因此受体 $3 \mathrm{c}$ 是一个理想的氟离子电化学传感分子.

\section{3 实验部分}

\section{1 试剂与仪器}

上海申光 WRS-1B 熔点仪, 温度未经校正; Bruker AVANCE III 400M 核磁共振仪 (400 MHz, TMS 为内标); Thermo-Finngan-LCQ 质谱仪; Spectrum 2000 傅立叶变 换红外光谱仪 ( $\mathrm{KBr}$ 压片); Elementar Vario MICRO 元素 分析仪; MERCURY CCD/AFC 衍射仪; CHI620C 电化学 工作站. 二茂铁甲基咪唑按照文献[15]方法制备. 其它 试剂均为市售分析纯，使用前经无水处理.

\section{2 实验方法}

3.2.1 1, $1^{\prime}-(1,2$-乙二基)-二(1-二茂铁甲基)味唑六氟 磷酸盐 $(\mathbf{3 a})$ 的合成

氮气保护下, 在 $250 \mathrm{~mL}$ 三口烧瓶中加入二茂铁甲 基咪唑 $1(530 \mathrm{mg}, 2.0 \mathrm{mmol})$ 于 $100 \mathrm{~mL}$ 的干燥 THF 中, 加入 1,2-二澳乙烷 $(180 \mathrm{mg}, 1.0 \mathrm{mmol})$ 后先室温搅拌 0.5 $\mathrm{h}$, 再加热回流 $24 \mathrm{~h}$, 得到稠状混合物. 将混合物溶于甲 醇溶液中, 加入 $\mathrm{NH}_{4} \mathrm{PF}_{6}(410 \mathrm{mg}, 2.5 \mathrm{mmol})$ 后室温继续 搅拌 $24 \mathrm{~h}$, 旋干溶剂, 用 $\mathrm{CH}_{2} \mathrm{Cl}_{2}$ 溶解, 水洗, 用无水硫 酸钠干燥, 碱性氧化铝柱层析得到 $686 \mathrm{mg}$ 黄色固体 3a, 
产率 82\%. m.p. 189 $191{ }^{\circ} \mathrm{C} ;{ }^{1} \mathrm{H}$ NMR $(400 \mathrm{MHz}$, DMSO- $\left.d_{6}\right) \delta: 8.98(\mathrm{~s}, 2 \mathrm{H}, \mathrm{NCHN}), 7.76(\mathrm{~s}, 2 \mathrm{H}, \mathrm{NCH})$, $7.58(\mathrm{~s}, 2 \mathrm{H}, \mathrm{NCH}), 5.12\left(\mathrm{~s}, 4 \mathrm{H}, \mathrm{CH}_{2}\right), 4.63\left(\mathrm{~s}, 4 \mathrm{H}, \mathrm{CH}_{2}\right)$, 4.40 (s, 4H, Cp-H), 4.27 (s, 4H, Cp-H), 4.25 (s, 10H, $\mathrm{Cp}-\mathrm{H}) ;{ }^{13} \mathrm{C}$ NMR (100 MHz, DMSO- $\left.d_{6}\right) \delta: 136.35$ (imi-C), 123.09 (NCH-C), 122.90 (NCH-C), 80.87 (Cp-C), 69.48 (Cp-C), 69.47 (Cp-C), 69.25 (Cp-C), $49.06\left(\mathrm{CH}_{2}-\mathrm{C}\right), 48.78$ $\left(\mathrm{CH}_{2}\right.$-C); IR (KBr) v: 3157, 3108, 1563, 1450, 1332, 1149 , 837, $557 \mathrm{~cm}^{-1}$; MS-ESI $m / z$ : $704.84\left[\mathrm{M}-\mathrm{PF}_{6}^{-}\right]^{+}$. Anal. calcd for $\mathrm{C}_{30} \mathrm{H}_{32} \mathrm{~F}_{12} \mathrm{Fe}_{2} \mathrm{~N}_{4} \mathrm{P}_{2}$ : C 42.38, H 3.79, N 6.59; found $\mathrm{C} 42.65, \mathrm{H} 3.69, \mathrm{~N} 6.66$.

3.2.2 1, $1^{\prime}-(1,3$-丙二基)-二(1-二茂铁甲基)咪唑六氟 磷酸盐 $(3 b)$ 的合成

合成方法同 3a, 黄色固体, 产率 80\%. m.p. 185 $186{ }^{\circ} \mathrm{C}$; ${ }^{1} \mathrm{H}$ NMR $\left(400 \mathrm{MHz}\right.$, DMSO- $\left.d_{6}\right) \delta$ : 9.09 (s, $2 \mathrm{H}$, $\mathrm{NCHN}$ ), 7.73 (d, $J=1.8,2 \mathrm{H}, \mathrm{NCH}), 7.69$ (d, $J=1.8,2 \mathrm{H}$, $\mathrm{NCH}$ ), 5.16 (s, 4H, $\mathrm{CH}_{2}$ ), 4.45 (s, 4H, Cp-H), 4.27 (s, 4H, Cp-H), 4.26 (s, 10H, Cp-H), 4.19 (t, J=7.2 Hz, 4H, $\mathrm{CH}_{2}$ ), $2.35\left(\mathrm{t}, J=7.2 \mathrm{~Hz}, 2 \mathrm{H}, \mathrm{CH}_{2}\right) ;{ }^{13} \mathrm{C}$ NMR $(100 \mathrm{MHz}$, DMSO- $d_{6}$ ) $\delta: 135.98$ (imi-C), 122.79 (NCH-C), 81.09 (Cp-C), 69.53 (Cp-C), 69.44 (Cp-C), 69.23 (Cp-C), 48.93 $\left(\mathrm{CH}_{2}-\mathrm{C}\right), 46.53\left(\mathrm{CH}_{2}-\mathrm{C}\right), 29.79\left(\mathrm{CH}_{2}-\mathrm{C}\right)$; IR ( $\left.\mathrm{KBr}\right) v: 3163$, $3113,1564,1450,1335,1151,841,559 \mathrm{~cm}^{-1}$; MS-ESI $m / z: 718.96\left[\mathrm{M}-\mathrm{PF}_{6}^{-}\right]^{+}$. Anal. calcd for $\mathrm{C}_{31} \mathrm{H}_{34} \mathrm{~F}_{12} \mathrm{Fe}_{2}-$ $\mathrm{N}_{4} \mathrm{P}_{2}$ : C 43.08, H 3.97, N 6.48; found C 43.25, H 4.18, N 6.64 .

3.2.3 1, $1^{\prime}-(1,4-$-丁二基)-二(1-二茂铁甲基)咪唑六氟 磷酸盐 $(3 c)$ 的合成

合成方法同 3a, 黄色固体, 产率 80\%. m.p. 134 $135{ }^{\circ} \mathrm{C}$; ${ }^{1} \mathrm{H}$ NMR $\left(400 \mathrm{MHz}\right.$, DMSO- $\left.d_{6}\right) \delta$ : 9.14 (s, $2 \mathrm{H}$, $\mathrm{NCHN}), 7.76(\mathrm{~d}, J=1.7 \mathrm{~Hz}, 2 \mathrm{H}, \mathrm{NCH}), 7.71$ (d, $J=1.7$ $\mathrm{Hz}, 2 \mathrm{H}, \mathrm{NCH}), 5.15$ (s, 4H, $\mathrm{CH}_{2}$ ), 4.44 (s, 4H, Cp-H), 4.26 (s, 4H, Cp-H), 4.24 (s, 10H, Cp-H), 4.20 (t, J=7.2 Hz, 4H, $\left.\mathrm{CH}_{2}\right), 1.74\left(\mathrm{t}, J=7.2 \mathrm{~Hz}, 4 \mathrm{H}, \mathrm{CH}_{2}\right) ;{ }^{13} \mathrm{C} \mathrm{NMR}(100 \mathrm{MHz}$, DMSO- $\left.d_{6}\right) \delta$ : 135.88 (imi-C), 122.82 (NCH-C), 81.31 (Cp-C), 69.43 (Cp-C), 69.39 (Cp-C), 69.21 (Cp-C), 55.37 $\left(\mathrm{CH}_{2}-\mathrm{C}\right), 48.73\left(\mathrm{CH}_{2}-\mathrm{C}\right), 48.59\left(\mathrm{CH}_{2}-\mathrm{C}\right), 26.64\left(\mathrm{CH}_{2}-\mathrm{C}\right)$; IR (KBr) v: 3169, 3105, 1564, 1446, 1155, 839, $557 \mathrm{~cm}^{-1}$; MS-ESI $m / z: 732.98\left[\mathrm{M}-\mathrm{PF}_{6}^{-}\right]^{+}$. Anal. calcd for $\mathrm{C}_{32} \mathrm{H}_{36}$ $\mathrm{F}_{12} \mathrm{Fe}_{2} \mathrm{~N}_{4} \mathrm{P}_{2}$ : C 43.76, H 4.13, N 6.38; found C 43.53, H $4.32, \mathrm{~N} 6.54$.

3.2.4 1, $1^{\prime}-(1,5$-戊二基)-二(1-二茂铁甲基)咪唑六氟 磷酸盐 $(3 d)$ 的合成

合成方法同 3a, 黄色固体, 产率 78\%. m.p. 79 $81{ }^{\circ} \mathrm{C} ;{ }^{1} \mathrm{H}$ NMR (400 MHz, DMSO- $d_{6}$ ) $\delta: 9.12$ (s, $2 \mathrm{H}$,
NCHN), 7.76 (d, $J=1.7 \mathrm{~Hz}, 2 \mathrm{H}, \mathrm{NCH}), 7.76(\mathrm{~d}, J=1.7$ $\mathrm{Hz}, 2 \mathrm{H}, \mathrm{NCH}), 5.16$ (s, 4H, $\left.\mathrm{CH}_{2}\right), 4.44$ (t, $J=1.8 \mathrm{~Hz}, 4 \mathrm{H}$, Cp-H), 4.26 (t, $J=1.8 \mathrm{~Hz}, 4 \mathrm{H}, \mathrm{Cp}-\mathrm{H}), 4.24$ (s, 10H, Cp-H), 4.15 (t, $J=7.6 \mathrm{~Hz}, 4 \mathrm{H}, \mathrm{CH}_{2}$ ), 1.79 (tt, $J=9.6,7.6 \mathrm{~Hz}, 4 \mathrm{H}$, $\left.\mathrm{CH}_{2}\right), 1.19$ (tt, $\left.J=9.6,7.6 \mathrm{~Hz}, 2 \mathrm{H}, \mathrm{CH}_{2}\right) ;{ }^{13} \mathrm{C}$ NMR $(100$ $\mathrm{MHz}, \mathrm{DMSO}-d_{6}$ ) $\delta: 135.79$ (imi-C), 122.85 (NCH-C), 122.75 (NHC-C), 81.36 (Cp-C), 69.41 (Cp-C), 69.40 (Cp-C), 69.21 (Cp-C), $55.37\left(\mathrm{CH}_{2}-\mathrm{C}\right), 48.93\left(\mathrm{CH}_{2}-\mathrm{C}\right)$, $29.12\left(\mathrm{CH}_{2}-\mathrm{C}\right), 22.63\left(\mathrm{CH}_{2}-\mathrm{C}\right)$; IR ( $\left.\mathrm{KBr}\right) v$ : 3162, 3103, 1564, 1467, 1151, 839, $557 \mathrm{~cm}^{-1}$; MS-ESI $\mathrm{m} / z: 747.00$ $\left[\mathrm{M}-\mathrm{PF}_{6}^{-}\right]^{+}$. Anal. calcd for $\mathrm{C}_{33} \mathrm{H}_{38} \mathrm{~F}_{12} \mathrm{Fe}_{2} \mathrm{~N}_{4} \mathrm{P}_{2}: \mathrm{C} 44.42$, H 4.29, N 6.28; found C 44.21, H 4.32, N 6.50.

$3.2 .51,1^{\prime}-(1,6$-已二基)-二(1-二茂铁甲基)咪唑六氟 磷酸盐 $(3 e)$ 的合成

合成方法同 3a, 黄色固体, 产率 78\%. m.p. 76 $78{ }^{\circ} \mathrm{C} ;{ }^{1} \mathrm{H}$ NMR (400 MHz, DMSO- $\left.d_{6}\right) \delta: 9.13$ (s, 2H, $\mathrm{NCHN}), 7.77(\mathrm{~d}, J=1.7 \mathrm{~Hz}, 2 \mathrm{H}, \mathrm{NCH}), 7.72(\mathrm{~d}, J=1.7$ $\mathrm{Hz}, 2 \mathrm{H}, \mathrm{NCH}), 5.16\left(\mathrm{~s}, 4 \mathrm{H}, \mathrm{CH}_{2}\right), 4.44(\mathrm{t}, J=1.8 \mathrm{~Hz}, 4 \mathrm{H}$, Cp-H), 4.26 (t, $J=1.8 \mathrm{~Hz}, 4 \mathrm{H}, \mathrm{Cp}-\mathrm{H}), 4.24(\mathrm{~m}, 10 \mathrm{H}$, Cp-H), 4.10 (t, $\left.J=7.2 \mathrm{~Hz}, 4 \mathrm{H}, \mathrm{CH}_{2}\right), 1.75$ (t, $J=7.2 \mathrm{~Hz}$, $\left.4 \mathrm{H}, \mathrm{CH}_{2}\right), 1.20 \sim 1.35\left(\mathrm{~m}, 4 \mathrm{H}, \mathrm{CH}_{2}\right) ;{ }^{13} \mathrm{C}$ NMR $(100 \mathrm{MHz}$, DMSO- $d_{6}$ ) $\delta: 135.79$ (imi-C), 122.85 (NCH-C), 122.75 (NHC-C), 81.36 (Cp-C), 69.41 (Cp-C), 69.40 (Cp-C), 69.21 (Cp-C), $55.37\left(\mathrm{CH}_{2}-\mathrm{C}\right), 48.93\left(\mathrm{CH}_{2}-\mathrm{C}\right), 29.12$ $\left(\mathrm{CH}_{2}-\mathrm{C}\right), 22.63\left(\mathrm{CH}_{2}-\mathrm{C}\right)$; IR (KBr) v: 3151, 2943, 1560, 1454, 1162, 1105, 842, $557 \mathrm{~cm}^{-1}$; MS-ESI $\mathrm{m} / z: 761.02$ $\left[\mathrm{M}-\mathrm{PF}_{6}^{-}\right]^{+}$. Anal. calcd for $\mathrm{C}_{34} \mathrm{H}_{40} \mathrm{~F}_{12} \mathrm{Fe}_{2} \mathrm{~N}_{4} \mathrm{P}_{2}: \mathrm{C} 45.06$, H 4.45, N 6.18; found C 45.29, H 4.63, N 6.26.

\subsection{6 化合物 $3 \mathrm{e}$ 的晶体结构测定}

通过石油梄扩散到乙腈溶液中得到化合物 $3 \mathrm{e}$ 单晶, 选取合适的晶体用 MERCURY CCD/AFC 衍射仪 100(2) $\mathrm{K}$ 下用石墨单色器单色化的 $\mathrm{Mo} \mathrm{K \alpha}$ 射线 $(\lambda=0.071073$ $\mathrm{nm})$ 作衍射光源, 以 $\omega$ 的扫描方式收集衍射点, 利用 $I>$ $2 \sigma(I)$ 独立衍射点用于晶体的结构分析. 采用 SHELXL-97 程序用直接法解出非氢原子坐标, 全矩阵 最小二乘法对非氢原子的坐标、各向异性、温度因子进 行修正 ${ }^{[16]}$.

\subsection{7 电化学滴定测试}

CHI620C 电化学工作站, 玻碳工作电极, 铂为对电 极, $\mathrm{Ag} / \mathrm{AgCl}$ (饱和 $\mathrm{KCl}$ ) 为参比电极. 乙腈溶液中测试, 受体 3a 3e $\left(10^{-3} \mathrm{~mol} \cdot \mathrm{L}^{-1}\right)$ 和支持电解质 $n-\mathrm{Bu}_{4} \mathrm{PF}_{6}(0.1$ $\mathrm{mol} \cdot \mathrm{L}^{-1}$ ) 在扫描速率 $100 \mathrm{mV} \cdot \mathrm{s}^{-1}$ 的条件下利用累积滴加 法将阴离子依次以不同物质的量滴入受体进行电化学 阴离子识别研究. 


\subsection{8 ${ }^{1} \mathrm{H}$ NMR 滴定测试}

Bruker AVANCE III 400M 核磁共振仪, DMSO- $d_{6}$ 溶 液中测试, 在受体 $3 \mathbf{a} \sim 3 \mathbf{e}\left(5 \times 10^{-4} \mathrm{~mol} \cdot \mathrm{L}^{-1}\right)$ 中利用累积 滴加法逐次加入四丁基铵盐的阴离子, 观察其受体分子 与阴离子结合后活泼氢化学位移的变化.

\section{辅助材料(Supporting Information) 受体分子 $\mathbf{3 a} \sim \mathbf{3 e}$} 的 NMR 谱图, 受体 $3 \mathrm{c}$ 在 DMSO- $d_{6}$ 中加入不同阴离子 的 ${ }^{1} \mathrm{H}$ NMR 堆积图, 受体分子 $\mathbf{3 e}$ 的氢键参数. 这些材料 可以免费从本刊网站(http://sioc-journal.cn/)上下载.

\section{References}

[1] (a) Maeda, H.; Shirai, T.; Bando, Y.; Takaishi, K.; Uchiyama, M.; Muranaka, A.; Kawai, T.; Naito, M. Org. Lett. 2013, 15, 6006.

(b) Hua, Y.; Flood, A. H. Chem. Soc. Rev. 2010, 39, 1262.

(c) Huang, Z. B.; Liu, X. C.; Hu, M. H.; Lin, W.; Shi, D. Q. Chin. J. Org. Chem. 2014, 34, 382 (in Chinese).

(黄志斌, 刘学成, 胡明华, 林伟, 史达清, 有机化学, 2014, 34, 382.)

[2] (a) Wade, C. R.; Broomsgrove, A. E.; Aldridge, S.; Gabbai, F. P. Chem. Rev. 2010, 110, 3958.

(b) Zhang, D. W.; Yang, H. Q.; Martinez, A.; Jamieson, K.; Dutasta, J. P.; Gao, G. H. Chem. Eur. J. 2014, 20, 1.

(c) Liu, R. Y.; Yong, X.; Yang, X. D.; Yan, Y. S.; Lu, X. W.; Qu, J. Q. Chin. J. Org. Chem. 2014, 34, 561 (in Chinese).

(刘瑞源, 勇雪, 杨小东, 严轶琛, 路新卫, 篗金清, 有机化学, 2014, 34, 561.)

[3] Beer, P. D.; Gale, P. A. Angew. Chem., Int. Ed. 2001, 40, 486.
[4] Niu, H. T.; Yin, Z.; Su, D.; Niu, D.; Ao, Y.; He, J.; Cheng, J. P. Tetrahedron 2008, 64, 6300 .

[5] Yoon, J.; Kim, S. K.; Singh, N. J.; Kim, K. S. Chem. Soc. Rev. 2006, 35, 355

[6] (a) Su, Z. M.; Ye, H. M.; Zhu, X. X.; Xie, L. L.; Bai, S.; Yuan, Y. F. J. Organomet. Chem. 2014, 750, 162.

(b) Zhuo, J. B.; Zhang, C. Y.; Lin, C. X.; Bai, S.; Xie, L. L.; Yuan, Y. F. J. Organomet. Chem. 2014, 763 764, 34

[7] Cao, Q. Y.; Han, Y. M.; Yao, P. S.; Fu, W. F.; Xie, Y.; Liu J. H. Tetrahedron Lett. 2014, 55, 248.

[8] (a) Beer, P. D.; Hayes, E. J. Coord. Chem. Rev. 2003, 240, 167. (b) Caballero, A.; Martínez, R.; Lloveras, V.; Ratera, I.; Vidal-Gancedo, J.; Wurst, K.; Tárraga, A.; Molina, P.; Veciana, J. J. Am. Chem. Soc. 2005, 127, 15666.

(c) Cao, Q. Y.; Zhang, J. F.; Ren, W. X.; Choi, K.; Kim, J. S. Tetrahedron Lett. 2011, 52, 4464.

[9] Zhuo, J. B.; Zhu, X. X.; Lin, C. X.; Bai, S.; Xie, L. L.; Yuan, Y. F. J. Organomet. Chem. 2014, 770, 85.

[10] Niu, H. T.; Yin, Z.; Su, D.; Niu, D.; He, J.; Cheng, J. P. Dalton Trans. 2008, 3694.

[11] Satheshkumar, A.; Manivannan, R.; Elango, K. P. J. Organomet. Chem. 2014, 750, 98.

[12] Klimova, T.; Klimova, E. I.; García, M. M.; Stivalet, J. M. M.; Ramírez, L. R. J. Organomet. Chem. 2001, 633, 137.

[13] Kong, D.; Weng, T.; He, W.; Liu, B.; Jin, S.; Hao, X.; Liu, S. J. Organomet. Chem. 2013, 727, 19.

[14] Thakur, A.; Mandal, D.; Sao, S.; Ghosh, S. J. Organomet. Chem. 2012, 715, 129.

[15] Thomas, J. L.; Howarth, J.; Hanlon, K.; McGuirk, D. Tetrahedron Lett. 2000, 41, 413.

[16] Sheldrick, G. M. Acta Crystallogr., Sect. A: Found. Crystallogr. 2008, 64, 112.

(Li, L.; Fan, Y.) 